\title{
Barium titanate nanoparticles used as second harmonic radiation imaging probes for cell imaging
}

\author{
Chia-Lung Hsieh*a,b, Rachel Grange ${ }^{\mathrm{a}}, \mathrm{Ye} \mathrm{Pu}^{\mathrm{a}, \mathrm{b}}$, Demetri Psaltis ${ }^{\mathrm{a}, \mathrm{b}}$ \\ ${ }^{a}$ School of Engineering, EPFL, Station 17, 1015 Lausanne, Switzerland; \\ ${ }^{\mathrm{b}}$ Department of Electrical Engineering, California Institute of Technology, 1200 East \\ California Boulevard, MC 136-93, Pasadena, California 91125, USA
}

\begin{abstract}
Luminescent markers play a key role in imaging techniques for life science since they provide a contrast mechanism between signal and background. We describe a new type of marker using second harmonic generation (SHG) from noncentrosymmetric $\mathrm{BaTiO}_{3}$ nanocrystals. These nanoparticles are attractive due to their stable, non-saturating and coherent signal with a femtosecond-scale response time and broad flexibility in the choice of excitation wavelength. In this paper, we report the use of nanoparticles for cell imaging. We first stabilized the $\mathrm{BaTiO}_{3}$ nanoparticles in suspension and characterized the optical properties. We also demonstrated the functionalization of $\mathrm{BaTiO}_{3}$ nanoparticles by conjugating $\operatorname{IgG}$ antibody on the surface of the nanoparticles. These functionalized nanocrystals are capable of specific labeling the antigens of interest.
\end{abstract}

Keywords: second harmonic generation, barium titanate, nanoparticles, biological cells, antibody

\section{INTRODUCTION}

Detecting and tracing specific molecules of interest is important in studying biology as it is closely connected to the function and the behavior of a biological system. Biomarkers have been developed to achieve this goal by creating a contrast between signal and background. An extremely effective method to obtain a contrast is to shift the signal away from the excitation wavelength so that the excitation can be efficiently removed by optical filters. Several mechanisms of converting the wavelength have been demonstrated, such as single-photon/two-photon fluorescence (1PF/2PF), second harmonic generation (SHG), and third harmonic generation (THG). Fluorescent biomarkers such as organic dyes [1], green fluorescent proteins (GFPs) [2], and quantum dots (QDs) [3] have been popularly used for biomedical imaging due to their excellent brightness and biocompatibility.

We have developed a new type of marker using the SHG from noncentrosymmetric nanocrystals which we refer to as "Second Harmonic Radiation IMaging Probes (SHRIMPs)". When a nanocrystal of noncentrosymmetric structure is optically excited at a fundamental frequency, it emits the optical signal at the exact doubled frequency. Only materials with crystalline structures lacking a center of symmetry are capable of efficient SHG. Thus, when imaged at the second harmonic frequency, SHRIMPs provide an effective mechanism of contrast between the markers and the generally unstructured or isotropic biological microenvironment. Ordered and highly polarizable biological noncentrosymmetric structures, such as the collagen fibers, have been known for endogenous SHG [4]. However, in most of the biological cell components, the endogenous SHG from the cell interface layers is weak [5].

Unlike fluorescence, the process of SHG only involves virtual electron energy transition without nonradiative energy loss. Owing to these lossless, virtual transition processes, SHG do not bleach over time and emit a stable, non-blinking signal that do not saturate over increasing excitation power. Furthermore, the response time of SHG process is in the femtosecond scale, which is four to five orders of magnitude faster than typical fluorophores. This allows for the observation of fast dynamic processes over a long time. In addition, SHG is generally a non-resonant process which offers the flexibility in the choice of excitation wavelength. This also results in the flexibility of tuning the wavelength of the SHG signal by changing the excitation wavelength accordingly. The coherent nature of the SHG signal is also a major advantage, providing a possibility to detect the second harmonic signal generated from the nanocrystals with interferometric optical techniques [6-8].

*chia-lung.hsieh@epfl.ch; phone +41 21693 7828; fax +41 21693 6930; http://lo.epfl.ch

Advanced Microscopy Techniques, edited by Paul J. Campagnola, Ernst H. K. Stelzer, Gert von Bally, Proc. of SPIE-OSA Biomedical Optics, SPIE Vol. 7367, 73670D · @ 2009 SPIE-OSA · CCC code: 1605-7422/09/\$18 · doi: 10.1117/12.831500 
The SHG properties of several kinds of nanocrystals have been recently reported: $\mathrm{BaTiO}_{3}[6,7], \mathrm{ZnO}[9,10], \mathrm{Fe}\left(\mathrm{IO}_{3}\right)_{3}$ [11], $\mathrm{KNbO}_{3}$ [12], $\mathrm{KTiOPO}_{4}$ (KTP) [8, 13] and SHG-active crystalline organic-inorganic hybrid nanoparticles [14]. For biomarkers applications, it is essential to prepare a uniform water dispersion of well-separated single nanocrystals and also a biological interface for the recognition and specific labeling. It has been reported that $\mathrm{ZnO}$ nanocrystals can be stabilized in aqueous suspension and further incorporated with folic acid molecules when being encapsulated in phospholipid micelles [9]. In this paper, we demonstrate the stabilization and functionalization of $\mathrm{BaTiO}_{3}$ nanoparticles, as well as conjugation of IgG antibody on the surface of the nanoparticles. The antibody conjugation provides a specific and flexible way to target any antigen on cells.

\section{STABILIZATION OF THE SHRIMP COLLOID}

We started the sample preparation from $90-\mathrm{nm} \mathrm{BaTiO}_{3}$ nanoparticles in dry powder which were commercially obtained from Techpowder. The X-ray diffraction measurement from the suppliers confirmed that the crystal structure is tetragonal, which is non-centrosymmetric, allowing for efficient SHG without any further treatment. Figure 1 (a) is the scanning electron microscope (SEM) image of the dry $\mathrm{BaTiO}_{3}$ nanoparticles showing nearly spherical shapes. The dry $\mathrm{BaTiO}_{3}$ nanoparticle powder was dispersed in $1.25 \mathrm{mM}$ aminomethylphosphonic acid with the particle concentration of $10^{10}$ particles $/ \mathrm{cm}^{3}$. We treated the colloidal suspension with ultrasound (Branson digital sonifier 450 ) at 50\% amplitude for 10 minutes to break the clusters into individual particles. The suspension was then stirred for 24 hours at $80^{\circ} \mathrm{C}$. After the treatment, the stability of the colloidal suspension was greatly improved. We washed the treated $\mathrm{BaTiO}_{3}$ nanoparticles with distilled water five times to remove the excess aminomethylphosphonic acid by using centrifugal filter devices (Amicon Ultra-15, 100KDa NMWL). Then, the particles were re-suspended in $\mathrm{pH} 8$ sodium hydroxide $(\mathrm{NaOH})$ in distilled water at the concentration of $5 \times 10^{11}$ particles $/ \mathrm{cm}^{3}$. The improvement of the colloidal stability is speculated as the result of the adsorption of the aminomethylphosphonic acid on the particle surface [15]. The zeta potential of the monodispersed colloidal suspension was obtained by dynamic light scattering measurement (Brookhaven Instruments Corp. ZetaPALS). We observed that the zeta potential decreases from $10 \mathrm{mV}$ to $-40 \mathrm{mV}$ when the $\mathrm{pH}$ of the solution increases from 2 to 9 . The size distribution of the treated $\mathrm{BaTiO}_{3}$ nanoparticles in suspension was measured by dynamic light scattering (Brookhaven Instruments Corp. 90Plus). As shown in Figure 1 (b), the $\mathrm{BaTiO}_{3}$ nanoparticles were well dispersed in water with a narrow size distribution around $90 \mathrm{~nm}$ (typical particle in the inset of Figure 1 (b)). The amine group adsorbed on the particle surface offers the general crosslink for further conjugation to proteins or antibodies for specific labeling [16].

(a)

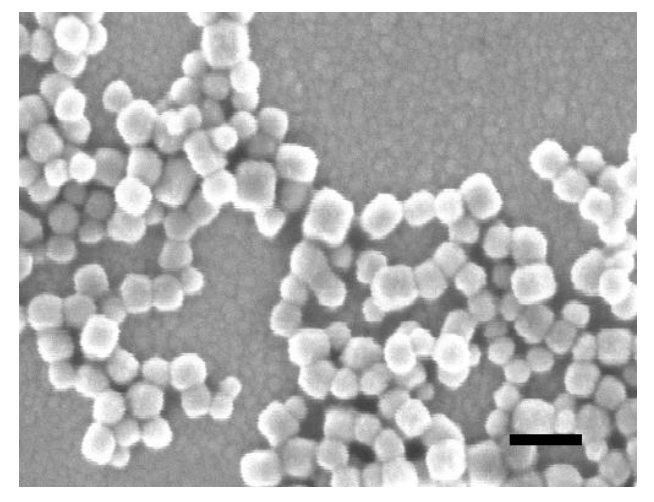

(b)

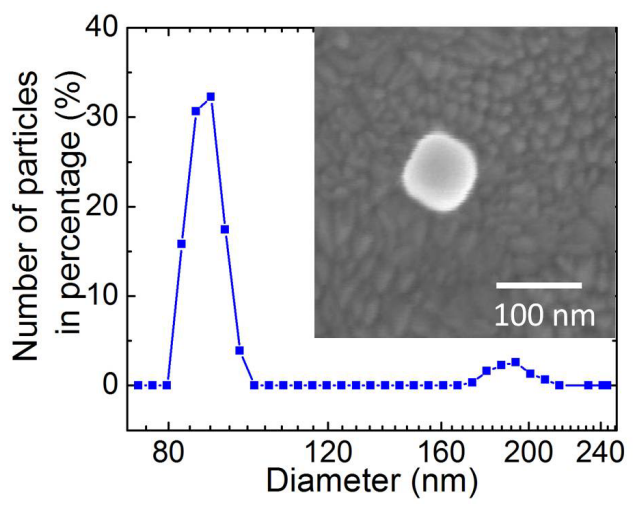

Fig. 1. (a) The SEM picture of $90-\mathrm{nm} \mathrm{BaTiO}_{3}$ nanocrystals. The scale bar is $200 \mathrm{~nm}$. (b) Dynamic light scattering measurement of $90-\mathrm{nm} \mathrm{BaTiO}{ }_{3}$ particles colloidal suspension. Inset: SEM image of a single $90-\mathrm{nm} \mathrm{BaTiO}_{3}$ particle.

\section{OPTICAL CHARACTERIZATION}

We characterized the SHG response of the nanoparticles which were dried on a glass substrate. A Ti:sapphire oscillator beam of $800 \mathrm{~nm}$ wavelength, $2 \mathrm{~nJ}$ pulse energy, $100 \mathrm{fs}$ pulse width, and $80 \mathrm{MHz}$ repetition rate was used for excitation. The laser beam was focused to reach the peak excitation intensity of $1 \mathrm{GW} / \mathrm{cm}^{2}$ on the sample. Figure 2 shows the second order nonlinear response of a $\sim 500-\mathrm{nm} \mathrm{BaTiO}_{3}$ cluster as a function of the excitation peak intensity up to 
$4 \mathrm{GW} / \mathrm{cm}^{2}$ at the sample position, which exactly follows a quadratic law. The inset of Fig. 2 shows the SHG optical spectrum centered around $400 \mathrm{~nm}$ with a FWHM of $5 \mathrm{~nm}$. We also recorded the SHG radiation over more than an hour without significant drop of the intensity.

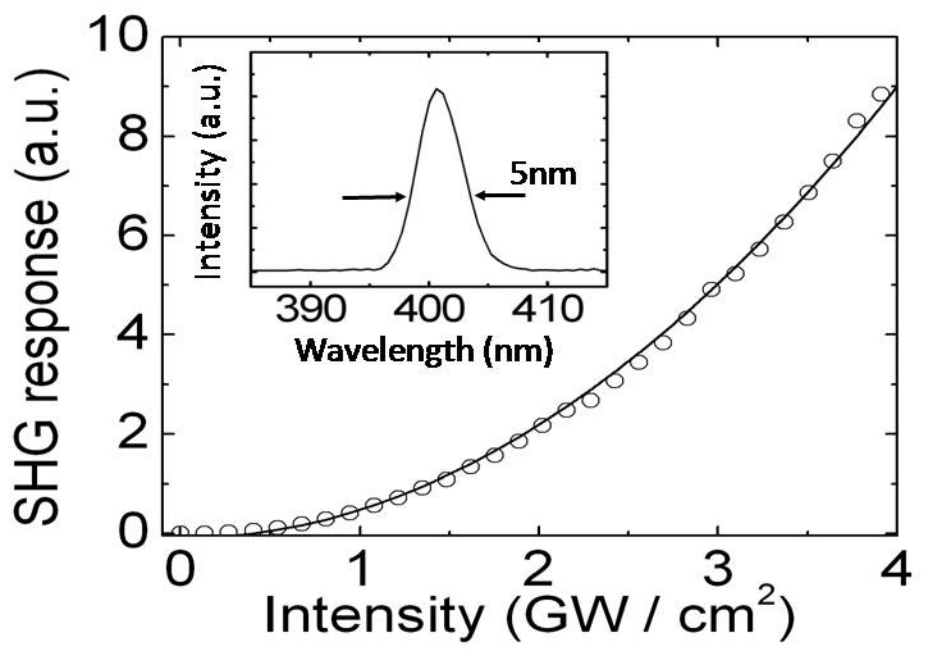

Fig. 2. Quadratic SHG response of $\mathrm{BaTiO}_{3}$ nanoparticles versus the excitation peak intensity. The circles show the experimental results while the line is the quadratic fit. Inset: optical spectrum of the SHG signal.

We used a two-photon laser scanning microscope (Leica, SP5) to image the dried 90-nm $\mathrm{BaTiO}_{3}$ nanoparticles on a glass substrate. The excitation laser of the microscope was a Ti:sapphire oscillator of $800 \mathrm{~nm}$ wavelength. Figure 3 (a) and (b) show the transmission and the $\mathrm{SHG}$ image of the $\mathrm{BaTiO}_{3}$ nanoparticles. In the transmission image, the nanoparticles scatter light, so they appear as dark spots. In the SHG image, the $\mathrm{BaTiO}_{3}$ nanoparticles give great contrast. Figure 3 shows a good correspondence of the transmission image to the SHG image. The intensity of the SHG signal from the nanoparticles varies, which may be due to the variation in particle size or to the polarization dependent SHG response. The second harmonic efficiency of a single $\mathrm{BaTiO}_{3}$ nanoparticle has been experimentally characterized and described with a model in reference 7 .

(a)

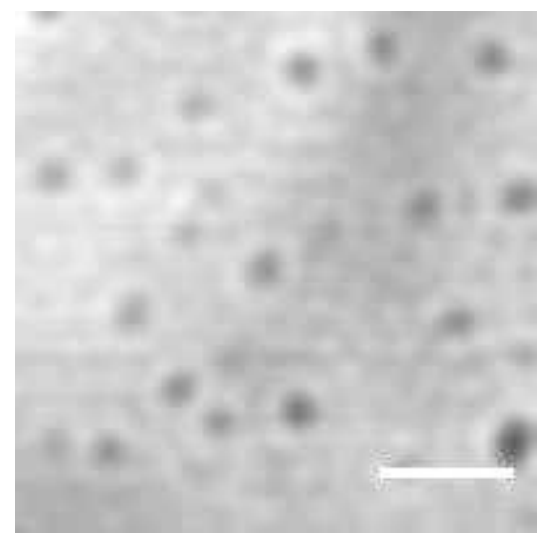

(b)

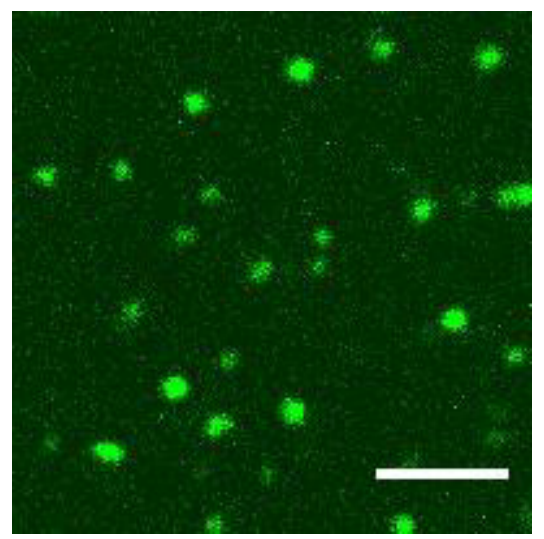

Fig. 3. Scanning images of $\mathrm{BaTiO}_{3}$ nanoparticles deposited on a glass substrate. (a) Transmission image. (b) SHG image. The scale bars are $5 \mu \mathrm{m}$.

\section{CELL IMAGING WITH SHRIMPS}

We demonstrate the capability of SHRIMPs as biomarkers for cell imaging. The sample was mammalian (HeLa) cells non-specifically labeled with SHRIMPs. The HeLa cells were incubated for 24 hours at $37^{\circ} \mathrm{C}$ with $\mathrm{BaTiO}_{3}$ particles that have been stabilized with aminomethylphosphonic acid. During the incubation, the SHRIMPs were uptaken by the cells through endocytosis [17]. SHRIMPs would be engulfed non-specifically into vesicles and packed as clusters randomly 
by the cells. After 24 hours incubation, the HeLa cells were still alive. We imaged the HeLa cells with SHRIMPs by a standard two-photon laser scanning microscope (Leica, SP5). The excitation laser was from a Ti:sapphire oscillator operated at $800 \mathrm{~nm}$. The HeLa cells were stained with Calcein so that we could see the cell through two-photon fluorescence signal from the dye. The SHG signal from SHRIMPs and the two-photon fluorescent signal from Calcein were collected simultaneously in two separated spectral channels. The section and cross section images of the HeLa cell with SHRIMPs are shown in Fig. 4. The SHRIMPs generated great contrast because the endogenous SHG from the cell interface layers was weak. Figure 4 confirms that the SHRIMPs entered the cells through endocytosis.
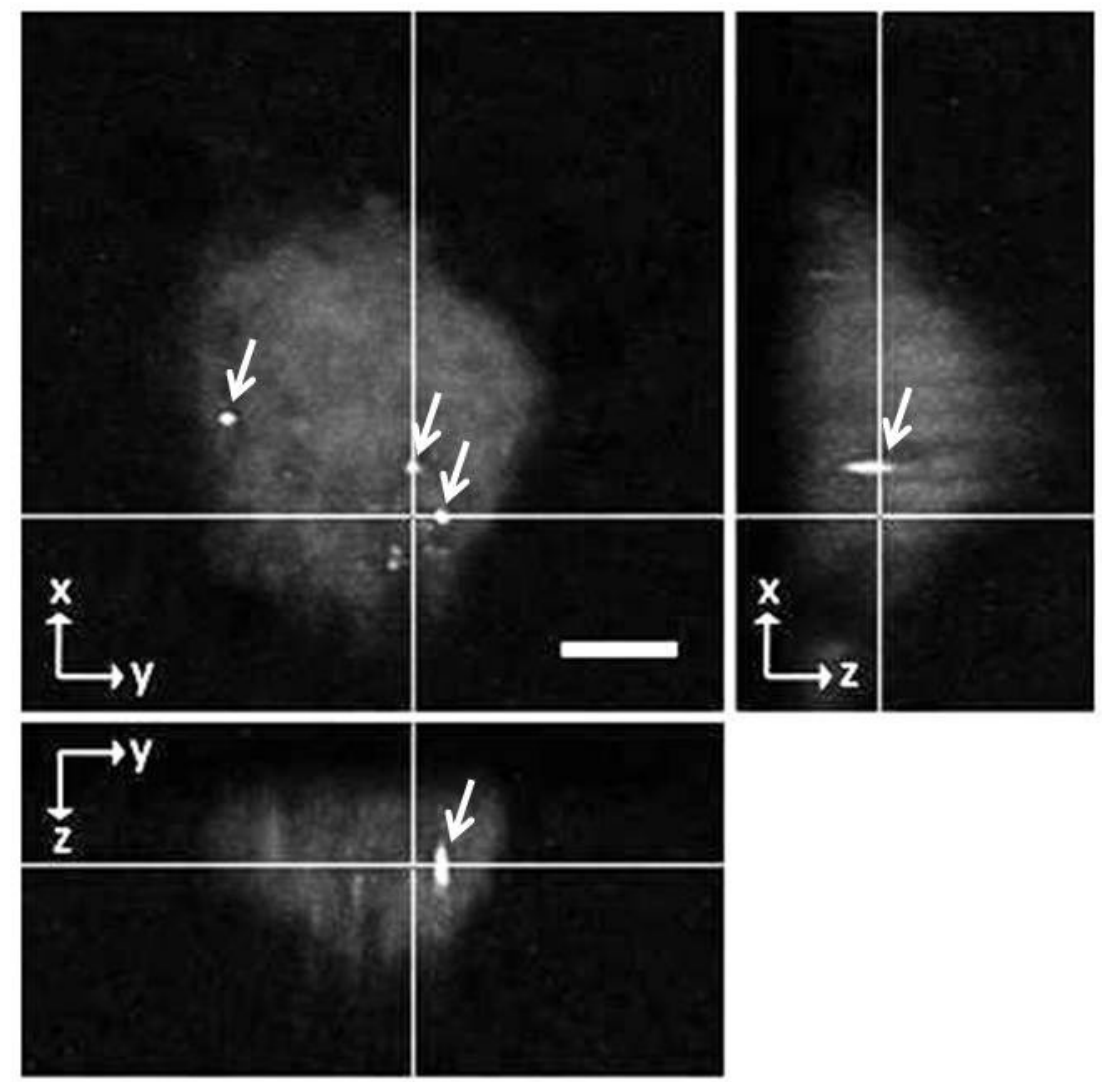

Fig. 4. Superimposed section images of the calcein-stained HeLa cell and SHRIMPs inside it. Calcein emits twophoton fluorescence signal and shows the shape of a HeLa cell. SHRIMPs inside the cell emit SHG signal as indicated by the white arrows in the image. It is clear that the SHRIMPs entered the cell through endocytosis. The scale bar is $5 \mu \mathrm{m}$.

\section{ANTIBODY CONJUGATION OF THE SHRIMPS}

To extract highly specific biological information requires that SHRIMPs be conjugated to functional biomolecules such as antibodies in order to target with specificity. The amine group adsorbed on the particle surface offers the general crosslink for further conjugation to proteins or antibodies for specific labeling. We conjugated HLA class I IgG antibodies to SHRIMPs based on crosslinking reactions between amine and sulfhydryl groups (catalyzed by maleimide, as shown in Fig. 5 (a)) [18]. The antibodies have fluorophores (FITC) on them which act as indicators of the presence of the antibodies. First, the amine groups on SHRIMPs reacted with the sulfo-SMCC (sulfosuccinimidyl 4-[Nmaleimidomethyl]cyclohexane-1-carboxylate) to yield maleimide-labeled SHRIMPs. Meanwhile, the hinge disulfide bonds on the IgG antibodies were reduced by DTT (dithiothreitol) to yield free sulfhydryl groups. The reduced antibodies were then coupled to the SHRIMPs through reaction of the maleimide groups with the free sulfhydryl on the IgG. 
We imaged the antibody-SHRIMP conjugates which were randomly attached to a glass substrate by the same two-photon scanning microscope described previously. The SHRIMPs emit SHG signal, while the fluorophores (FITC) on the antibodies emit two-photon fluorescence signal. These two signals were collected in two separate channels. Scanning images of the antibody-SHRIMP conjugates show a high correlation between the SHG signal from the SHRIMPs (Fig. 5 (b)) and the fluorescence signal from the FITC attached to the antibodies (Fig. 5 (c)). This result indicates the collocation of the antibodies and the SHRIMPs and therefore confirms the adsorption of the antibodies on the SHRIMPs. These antibody-SHRIMP conjugates are ready to label specific antigen recognized by the antibody.

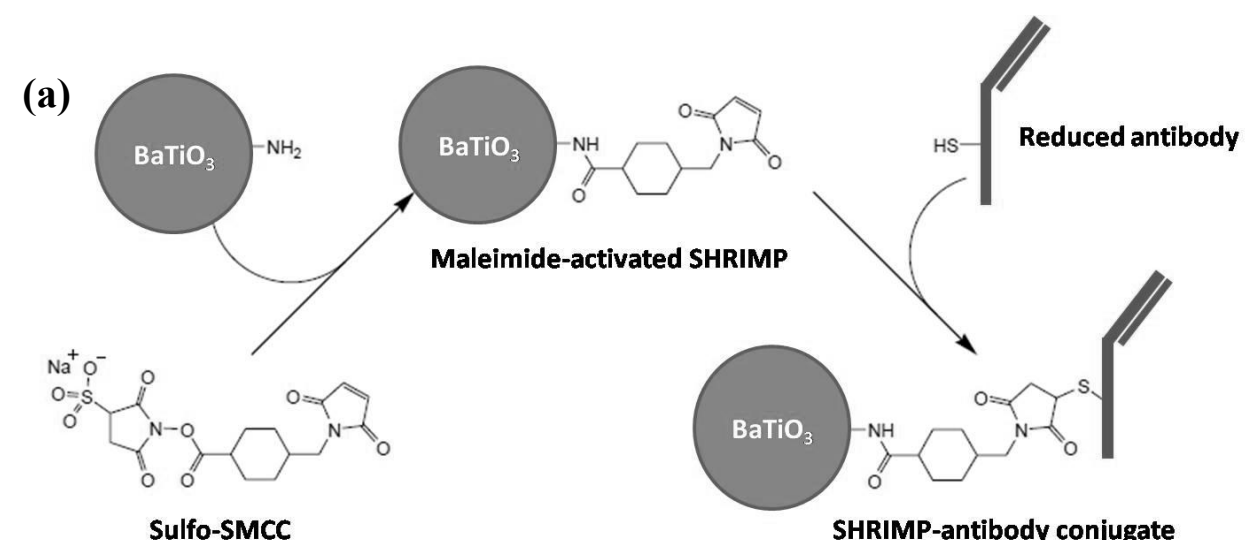

(b)
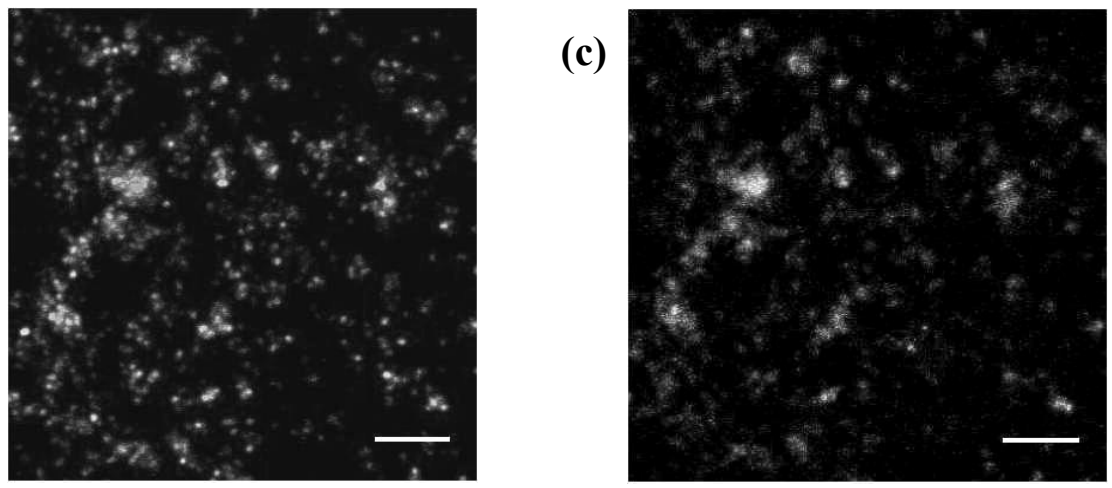

Fig. 5. The antibody-SHRIMP conjugations. (a) The illustrative diagram of conjugating the antibody to the SHRIMP. (b) The SHG images from the SHRIMPs randomly attached to a glass substrate. (c) The twophoton fluorescence image from the fluorophores FITC present on the IgG antibodies. (b) and (c) show great correlation, which indicates the collocation of the SHRIMPs and the antibodies. The scale bars are $5 \mu \mathrm{m}$.

\section{CONCLUSION}

We demonstrated the use of $\mathrm{BaTiO}_{3}$ nanoparticles as SHRIMPs for bio-imaging. We described the stabilization of the $\mathrm{BaTiO}_{3}$ nanoparticles in suspension and the characterization the optical properties. We also demonstrated the functionalization of $\mathrm{BaTiO}_{3}$ nanoparticles by conjugating IgG antibody on the surface of the nanoparticles. These functionalized nanocrystals are ready for specific labeling in biomedical applications. SHRIMPs possess several key advantages, owing to the intrinsic properties of SHG, including long-term observation without photobleaching, flexibility in excitation wavelength, coherent signals for three-dimensional imaging, narrow signal bandwidth for greater noise rejection, ultrafast response time, and excellent biocompatibility. In general, SHRIMPs are not replacements for fluorescent markers. Instead they enable new important functionalities that are not available with current techniques in a 
wide variety of applications. These unique properties will likely enable new microscopy technologies that are currently not accessible, providing means to extract more accurate and reliable biomedical information through imaging.

\section{REFERENCES}

[1] Griffin, B. A., Adams, S. R., and Tsien, R. Y., "Specific covalent labeling of recombinant protein molecules inside live cells," Science 281, 269-272 (1998).

[2] Chalfie, M., Tu, Y., Euskirchen, G., Ward, W. W., and Prasher, D. C.,"Green fluorescent protein as a marker for gene-expression," Science 263, 802-805 (1994).

[3] Chan, W. C. W., and Nie, S. M., "Quantum dot bioconjugates for ultrasensitive nonisotopic detection," Science 281, 2016-2018 (1998).

[4] Campagnola, P. J., and Loew, L. M., "Second-harmonic imaging microscopy for visualizing biomolecular arrays in cells, tissues and organisms," Nat Biotech 21, 1356-1360 (2003).

[5] Campagnola, P. J., Wei, M. D., Lewis, A., and Loew, L. M., "High-resolution nonlinear optical imaging of live cells by second harmonic generation," Biophys. J. 77, 3341-3349 (1999).

[6] Pu, Y., Centurion, M., and Psaltis, D., "Harmonic holography: a new holographic principle," Appl. Opt. 47, A103A110 (2008).

[7] Hsieh, C.-L., Grange, R., Pu Y., Psaltis, D., "Three-dimensional harmonic holographic microscopy using nanoparticles as probes for cell imaging," Opt. Express 17, 2880-2891 (2009).

[8] Xuan, L. L., Brasselet, S., Treussart, F., Roch, J. F., Marquier, F., Chauvat, D., Perruchas, S., Tard, C., and Gacoin, T., "Balanced homodyne detection of second-harmonic generation from isolated subwavelength emitters," Appl. Phys. Lett. 89, 121118 (2006).

[9] Kachynski, A. V., Kuzmin, A. N., Nyk, M., Roy, I., and Prasad, P. N., "Zinc oxide nanocrystals for nonresonant nonlinear optical microscopy in biology and medicine," J. Phys. Chem. C 112, 10721-10724 (2008).

[10] Johnson, J. C., Yan, H. Q., Schaller, R. D., Petersen, P. B., Yang, P. D., and Saykally, R. J., "Near-field imaging of nonlinear optical mixing in single zinc oxide nanowires," Nano Letters 2, 279-283 (2002).

[11] Bonacina, L., Mugnier, Y., Courvoisier, F., Le Dantec, R., Extermann, J., Lambert, Y., Boutou, V., Galez, C., and Wolf, J. P., "Polar Fe(IO3)(3) nanocrystals as local probes for nonlinear microscopy," Appl. Phys. B-Lasers and Optics 87, 399-403 (2007).

[12] Nakayama, Y., Pauzauskie, P. J., Radenovic, A., Onorato, R. M., Saykally, R. J., Liphardt, J., and Yang, P. D., "Tunable nanowire nonlinear optical probe," Nature 447, 1098-1101 (2007).

[13] Sandeau, N., Le Xuan, L., Chauvat, D., Zhou, C., Roch, J. F., and Brasselet, S., "Defocused imaging of second harmonic generation from a single nanocrystal," Opt. Express 15, 16051-16060 (2007).

[14] Delahaye, E., Tancrez, N., Yi, T., Ledoux, I., Zyss, J., Brasselet, S., and Clement, R., "Second harmonic generation from individual hybrid MnPS3-based nanoparticles investigated by nonlinear microscopy," Chem. Phys. Lett. 429, 533-537 (2006).

[15] Kim P., Jones S. C., Hotchkiss P. J., Haddock J. N., Kippelen B., Marder S. R., and Perry J. W., "Phosphonic acidmodified barium titanate polymer nanocomposites with high permittivity and dielectric strength," Adv. Mater. 19, 1001-1005 (2007).

[16] Dubertret, B., Skourides, P., Norris, D. J., Noireaux, V., Brivanlou, A. H., and Libchaber, A., "In vivo imaging of quantum dots encapsulated in phospholipid micelles," Science 298, 1759-1762 (2002).

[17] Lorenz, M. R., Holzapfel, V., Musyanovych, A., Nothelfer, K., Walther, P., Frank, H., Landfester, K., Schrezenmeier, H., and Mailander, V., "Uptake of functionalized, fluorescent-labeled polymeric particles in different cell lines and stem cells," Biomaterials 27, 2820-2828 (2006).

[18] Xing, Y., Chaudry, Q., Shen, C., Kong, K. Y., Zhau, H. E, Chung, L. W, Petros, J. A, O'Regan, R. M, Yezhelyev, M. V, Simons, J. W, Wang M. D, and Nie, S., "Bioconjugated quantum dots for multiplexed and quantitative immunohistochemistry," Nat. Protocols 2, 1152-1165 (2007). 